\title{
The impact of disease activity and psychological status on quality of life for Chinese patients with primary Sjögren's syndrome
}

This article was published in the following Dove Press journal:

Patient Preference and Adherence

\author{
Yafei Cui ${ }^{1-3, *}$ \\ Lin $\mathrm{Li}^{2,4, *}$ \\ Ling Xial,* \\ Qian Zhao ${ }^{2}$ \\ Shengnan Chen ${ }^{2}$ \\ Ting Fu' \\ Juan Ji \\ Zhifeng Gu' \\ 'Department of Rheumatology, \\ Affiliated Hospital of Nantong \\ University, Nantong, China; ${ }^{2}$ School \\ of Nursing, Nantong University, \\ Nantong, China; ${ }^{3}$ Department \\ of Nursing, The First Affiliated \\ Hospital of Zhengzhou University, \\ Zhengzhou, China; ${ }^{4}$ Department of \\ Nursing, Changzhou 2nd People's \\ Affiliated Hospital of Nanjing Medical \\ University, Changzhou, China \\ *These authors contributed equally \\ to this work
}

Objective: The primary purpose of the present study was to survey the quality of life (QoL) in primary Sjögren's syndrome (pSS) and to analyze the relationships between disease activity, anxiety/depression, fatigue, pain, age, oral disorders, impaired swallowing, sicca symptoms, and QoL.

Patients and methods: A survey was conducted on 185 pSS patients and 168 healthy individuals using the Short Form 36 health survey for QoL. Disease activity was assessed using the European League Against Rheumatism Sjögren's Syndrome Disease Activity Index. We examined these data using independent samples $t$-tests, Mann-Whitney U test, chi squared analysis, and linear regression.

Results: The result for each domain in Short Form 36 health survey was lower in pSS patients than in healthy controls, especially the score in the dimension of role physical function. In the bivariate analysis, age, pain, fatigue, disease activity, disease complication, anxiety/depression, oral disorders, and impaired swallowing correlated with QoL. Also, in the linear regression model, pain, fatigue, disease activity, impaired swallowing, and anxiety/depression remained the main predictors of QoL.

Conclusion: pSS patients had a considerably impaired QoL compared to the controls, and pSS could negatively affect the QoL of patients. Measuring QoL should be considered as a vital part of the comprehensive evaluation of the health status of pSS patients, which could contribute some valuable clues in improving the management of disease and treatment decisions.

Keywords: primary Sjögren's syndrome, quality of life, disease activity, depression

\section{Background}

Primary Sjögren's syndrome (pSS) is a systemic autoimmune disease characterized by chronic inflammation of exocrine glands such as the lachrymal and salivary glands, leading to dry eyes and dry mouth. ${ }^{1}$ A majority of pSS patients also suffer from extraglandular symptoms including fatigue, mental disorders, musculoskeletal, and neurological symptoms. ${ }^{2}$ In addition, pSS patients exhibit a 44 times higher relative risk for lymphoma development compared to the general population. ${ }^{3}$ This disease predominantly affects middle-aged women (women:men ratio 9:1), but can also be detected in children and the aged. ${ }^{4} \mathrm{pSS}$ is a common autoimmune rheumatic condition, second only in frequency to rheumatoid arthritis, with a prevalence between $0.1 \%$ and $4.8 \%$ in various populations. ${ }^{5}$ pSS has a negative influence on health-related quality of life (HR-QoL) ${ }^{6}$ and is associated with high health care costs, ${ }^{7}$ pain, ${ }^{8}$ fatigue, ${ }^{9}$ work disability, ${ }^{10}$ and anxiety/depression. ${ }^{11}$ Moreover, dry mouth in pSS may lead to oral
Correspondence: Zhifeng Gu Department of Rheumatology, Affliated Hospital of Nantong University, 20th Xisi road, 22600 I Nantong, China

Tel +86 I370629 194I

Email guzf@ntu.edu.cn 
uncomfortableness, trouble talking, impaired swallowing, tooth decay, and continual oral infections. Though these complications are not life-threatening, the complication and chronicity of symptoms can lead to heavy debility and a reduction in patient's quality of life (QoL). ${ }^{12}$ Individuals with pSS also experience voice disturbances and specific voicerelated manifestations that are related to decreased QoL. ${ }^{13}$

QoL is defined as the general well-being of individuals and societies, based on the individual's culture and life values with respect to that individual's objectives, expectation, and standards. HR-QoL is the impact of disease and its treatment of the individual's ability to function based on physical, mental, and social well-being. ${ }^{14}$ Variables that may affect the QoL of pSS patients include disease activity, fibromyalgia, ${ }^{15}$ comorbidity, ${ }^{2}$ medical therapy, ${ }^{16}$ pain, fatigue, dryness, and anxiety/depression. In the literature, there are various studies investigating fatigue and discomfort, oral HR-QoL, ${ }^{17}$ employment and disability, ${ }^{18}$ and psychological status of pSS patients. However, to our best knowledge, there are a few previous studies focusing on how these variables might affect the HR-QoL in pSS, especially in China. The objective of the present study was to evaluate QoL in pSS and to explore the relationships between disease activity, fatigue, anxiety/depression, oral disorders, impaired swallowing, pain, age, dryness, and QoL.

\section{Patients and methods}

\section{Participants}

The study was carried out in the Affiliated Hospital of Nantong University from July 2016 to July 2017.pSS patients were outpatients or inpatients from the Department of Rheumatology; age- and sex-matched controls were randomly selected from the general population. The Ethics Committee of the Affiliated Hospital of Nantong University approved this study (approval number 2017-K003), and all participants met the American-European Consensus Group Criteria for pSS and gave written informed consent. All study participants were asked to complete the related questionnaires. Medical assessments also were recorded. There was no significant difference between the two groups in terms of age, sex, education level, occupation, and income/year.

\section{Inclusion criteria and exclusion criteria}

Inclusion criteria consisted of pSS diagnosis, age over 18 years, no known cognitive deficits, and Chinese speaking. Exclusion criteria consisted of secondary Sjögren syndrome, having serious systemic diseases lately (lymphoma, similar renal/pulmonary involvement, myositis, or vasculitis), and participants with a physical or psychological problem (eg, cancer, psychiatric disorders) which may confound the outcome.

\section{Methods}

This study included $185 \mathrm{pSS}$ patients according to the American-European Consensus Group criteria and 168 healthy controls. Sicca symptoms were assessed by the dryness domain of the European League Against Rheumatism Sjögren's Syndrome Patient-Reported Index. ${ }^{19}$ Disease activity was assessed using the European League Against Rheumatism Sjögren's Syndrome Disease Activity Index (ESSDAI) ${ }^{20}$ Mental state was assessed through Hospital Anxiety and Depression Scale; it had acceptable internal consistency and test-retest reliability, with a Cronbach's alpha of 0.85 and intraclass correlation coefficient of $0.90 .^{21}$ Independent samples $t$-tests, chi-squared analyses, MannWhitney $U$ test, and linear regression modeling were used to analyze the data.

\section{Demographics and clinical characteristics}

Demographic characteristics and clinical variables were examined. Demographic variables included age, sex, education, marital status, and socioeconomic status. Next, differences in mean Hospital Anxiety and Depression Scale scores and QoL scores between pSS patients and control groups were analyzed. Clinical data such as disease duration and medication use were obtained by asking the patients or searching their electronic medical records.

\section{The medical outcomes study, 36-item short-form health survey (SF-36)}

The SF-36 instrument is aimed to rate HR-QoL within the previous 4 weeks. It includes 36 statements, with eight scales assessing two dimensions. The first dimension is physical health function and includes the following four specific scores: physical functioning (the extent to which health interferes with various activities), physical role functioning (the extent to which health interferes with usual daily activities), bodily pain, and general health. These physical scores are summarized by the physical composite score (PCS). The second dimension is the mental health function, which includes the following four specific scores: vitality, social functioning, emotional role functioning (limitations due to emotional problems), and mental health. These four mental scores are summarized by the mental composite score 
(MCS). Each scale gives a standardized score that ranges from 0 to 100 , with 0 illustrating the worst possible health state and 100 illustrating the best possible health state. ${ }^{22,23}$ In addition, this scale has been translated and validated for use in Hong Kong. ${ }^{24,25}$

Convergent validity and discriminant validity were satisfactory for all except the social functioning scale. Cronbach's $\alpha$ coefficients ranged from 0.72 to 0.88 except 0.39 for the social functioning scale and 0.66 for the vitality scale. Two weeks test-retest reliability coefficients ranged from 0.66 to $0.94 .^{26}$

\section{Fatigue severity scale}

Fatigue was measured by using the fatigue severity scale. It is a self-reported instrument developed to explore the impact and severity of fatigue. It involves nine questions to assess the severity of fatigue, as it relates to daily activities such as physical functioning, exercise, work, and family/social life. The scores range from 1 to 7 for each item, with a lower score signifying less fatigue (the best condition is 9 points and the worst condition is 63 points). We used a cut-off score $\geq 4$ to define fatigue. ${ }^{27}$

\section{Other related scales}

Pain was investigated by the visual analog scale to identify pain severity. Patients were asked to estimate their experiences of pain during the last week, each on a visual analog scale of $0-10$, with a higher score implying more severe pain. ${ }^{28}$ The MD Anderson Dysphagia Inventory ${ }^{29}$ measures patient's swallowing disorders symptoms (score ranges from 0 to 100 , with higher scores indicating more severe symptoms), and the Oral Health Impact Profile $14^{30}$ was used to measure oral distress.

\section{Data analysis}

Data were analyzed by IBM SPSS 22.0 package program. Continuous variables were expressed as mean \pm SD. Before the continuous variables were compared, it was checked whether the parametric test assumptions were met or not. Qualitative variables were presented as numbers and percentages. The distinction between independent groups in terms of the qualitative variables was evaluated by using chi-squared test. While conformity to normal distribution was evaluated by using Shapiro-Wilk test, the homogeneity of the variances was examined by using the Levene's test. The difference between independent groups in terms of numerical variables was investigated by using the independent samples $t$-test in cases where the parametric test assumptions were met and by using the Mann-Whitney $U$ test in cases where the said assumptions were not met. The presence of a correlation between the numerical variables was determined by Spearman's correlation coefficient. Multicollinearity between variables was checked by computing variance inflation factors before inclusion into the model. A backward procedure was used, removing variables with a $P$-value of $>0.05$ in the multivariable model in order of significance, until the best-fitting model was identified. While we regarded $P$-values $<0.05$ in each study as significant, we set stringent significant levels of $P$-values in the combined analysis based on Bonferroni's correction.

\section{Written informed consent}

Written informed consent was obtained from all individual participants included in the study.

\section{Results}

\section{Patient characteristics}

Of the 203 eligible cases, 185 patients fully completed the provided questionnaires, with a rough response rate of $91 \%$. In $\mathrm{pSS}$ patients $(\mathrm{n}=185)$, the mean age was $50.12 \pm 12.13$ years, and $94.6 \%$ of the subjects were women. Demographic, clinical, and laboratory characteristics of the pSS patients and controls are presented in Table 1. Furthermore, the impact of disease-related variables on severity of depression and anxiety was assessed. Whether depression, anxiety, and diseaserelated variables can be determinants of QoL among affected individuals was also investigated. There was no significant difference in age, sex, education, occupation, and income/ year between the pSS individuals and the controls $(P>0.05)$. Tests of collinearity indicated good content validity.

\section{QoL of pSS patients and controls}

The scores of global SF-36, including PCS and MCS of pSS patients and the controls, are presented in Figure 1. Eight domains of SF-36 of the pSS patients and controls are shown in Table 2. The average global SF-36 score of pSS patients was $56.01 \pm 18.13$, ranging from 13.94 to 98.13 . Compared with the controls, all SF-36 domains and total scores were much lower in pSS patients $(P<0.01)$.

\section{Correlations between fatigue, disease activity etc, and QoL in PSS patients}

As shown in Table 3, we found signifcant correlations between erythrocyte sedimentation rate, comorbidity, 
Table I Demographic, clinical, and psychological characteristics of the pSS patients and controls

\begin{tabular}{|c|c|c|c|c|}
\hline Clinical characteristics & $\begin{array}{l}\text { pSS } \\
(n=185)\end{array}$ & $\begin{array}{l}\text { Controls } \\
(n=168)\end{array}$ & $t / \chi^{2} / z$ & $P$-value \\
\hline Age, mean $\pm S D$, years & $50.12 \pm 12.13$ & $50.25 \pm 11.17$ & -0.105 & 0.916 \\
\hline Sex, female, n (\%) & $175(94.6)$ & $160(95.2)$ & 0.075 & 0.784 \\
\hline Marital status, n (\%) & & & 5.590 & 0.018 \\
\hline Married & $172(93)$ & $165(98.2)$ & & \\
\hline Single/divorced/bereaved & $13(7)$ & $3(1.8)$ & & \\
\hline Education, mean $\pm S D$, years & $12.44 \pm 4.19$ & $11.99 \pm 4.64$ & 0.945 & 0.345 \\
\hline Employment status, n (\%) & & & 0.931 & 0.335 \\
\hline Employed & $83(44.9)$ & $84(50)$ & & \\
\hline Unemployed & $102(55.1)$ & $84(50)$ & & \\
\hline Income/person/year, yuan, n (\%) & & & $-0.67 \mid$ & 0.502 \\
\hline$<15,000$ yuan & $55(29.7)$ & $49(29.2)$ & & \\
\hline $15,000-33,000$ yuan & $55(29.7)$ & $61(36.3)$ & & \\
\hline$>33,000$ yuan & $75(40.5)$ & $58(34.5)$ & & \\
\hline Type of medical insurance, $\mathrm{n}(\%)$ & & & 3.582 & 0.167 \\
\hline With basic medical insurance & | 70 (9|.9) & $164(96.5)$ & & \\
\hline Self-pay & $14(7.6)$ & $6(3.6)$ & & \\
\hline Other & $\mathrm{I}(0.5)$ & $0(0)$ & & \\
\hline HADS-A, mean $\pm S D$ & $6.43 \pm 3.99$ & $3.04 \pm 2.78$ & 9.315 & 0.000 \\
\hline HADS-D, mean $\pm S D$ & $6.63 \pm 4.16$ & $3.89 \pm 2.8$ & 7.318 & 0.000 \\
\hline Oral disorders, n (\%) & $165(89.2)$ & $101(60.1)$ & 8.246 & 0.000 \\
\hline Impaired swallowing, mean $\pm S D$ & $75.52 \pm 18.04$ & - & & \\
\hline Disease duration, mean $\pm S D$, years & $4.34 \pm 5.24$ & - & & \\
\hline VAS pain (range $0-10$ ), mean $\pm S D$ & $2.15 \pm 2.56$ & - & & \\
\hline $\mathrm{ESR}$, mean $\pm \mathrm{SD} \mathrm{mm} / \mathrm{h}$ & $29.16 \pm 23.99$ & - & & \\
\hline Hospitalization, yes, n (\%) & $124(67)$ & - & & \\
\hline Comorbid conditions, yes, n (\%) & $112(60.5)$ & - & & \\
\hline ESSDAI, mean \pm SD & $9.56 \pm 7.3$ & - & & \\
\hline ESSPRI, mean \pm SD & $4.18 \pm 1.89$ & - & & \\
\hline Impaired swallowing, n (\%) & $51(27.57)$ & - & & \\
\hline Medicine use, n (\%) & $153(82.7)$ & - & & \\
\hline Glucocorticoids, yes, n (\%) & $56(30.3)$ & - & & \\
\hline Hydroxychloroquine, yes, n (\%) & $140(75.7)$ & - & & \\
\hline Others, yes, n (\%) & I 43 (77.3) & - & & \\
\hline
\end{tabular}

Abbreviations: ESR, erythrocyte sedimentation rate; ESSPRI, European League Against Rheumatism Patient Reported Index; ESSDAI, European League Against Rheumatism Sjögren's Syndrome Disease Activity Index; HADS, Hospital Anxiety and Depression Scale; HADS-A, Hospital Anxiety Scale; HADS-D, Hospital Depression Scale; pSS, primary Sjögren's syndrome; VAS, visual analog scale.

hospitalization, and European League Against Rheumatism Sjögren's Syndrome Patient-Reported Index and QoL.

\section{Stepwise linear regression analysis for the QoL}

Stepwise linear regression analysis revealed depression, pain, and impaired swallowing were notably associated with the SF-36 PCS in pSS patients. Moreover, depression, anxiety, disease activity, fatigue, and impaired swallowing significantly accounted for the SF-36 MCS (Table 4). Finally, these variables explained $65.5 \%$ and $72.5 \%$ of the variance in PCS and MCS, respectively.

\section{Discussion}

pSS patients experience lower QoL compared to ageand sex-matched controls. Impaired QoL is associated with symptoms such as fatigue, pain, anxiety/depression, oral disorders, impaired swallowing, as well as disease activity, exemplifying the value of optimal management of all aspects of the disease. The underlying pathogenic process between depression and $\mathrm{pSS}$ remains unknown. Some studies attempted to explore depression in pSS patients; depression was reported as a relative factor of reduced QoL in Moroccan pSS patients. ${ }^{31}$ Segal et al ${ }^{32}$ stated that somatic fatigue was the main predictor of physical function and general health; additionally, depression was the key predictor of emotional 


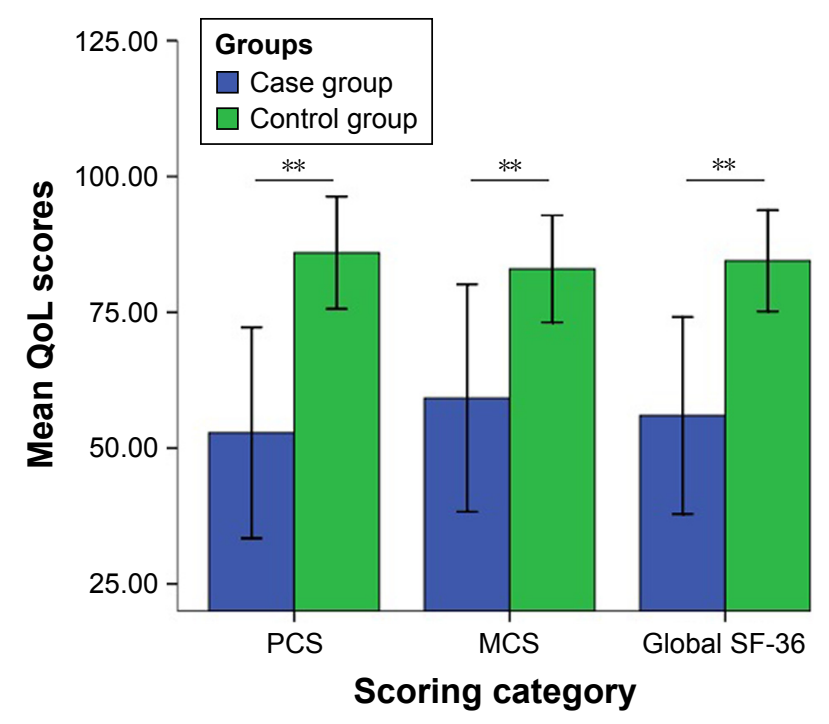

Figure I Global SF-36, PCS, and MCS among PSS patients and controls. Note: $* * P<0.01$.

Abbreviations: MCS, mental composite score; PCS, physical composite score pSS, primary Sjögren's syndrome; QoL, quality of life; SF-36, Short Form 36 health survey.

well-being in the US. In agreement with the findings of previous studies, our study found that the strong correlation between MCS of the SF-36 and anxiety/depression in pSS patients emphasized the significance of the psychological dimension of SF-36. Consequently, recognition of determinants of depression and anxiety and their impact on QoL has noticeable clinical value, especially in China.

Fatigue is another essential symptom and has been reported to be associated with a reduction in HR-QoL in pSS. ${ }^{33}$ A study from Germany reported that patients with pSS frequently suffer from fatigue that increases the risk of work disability and requires treatment by numerous health care specialists. ${ }^{34}$ As expected, bivariate analysis showed that

Table 2 Eight domains in SF-36 of patients with pSS and the controls

\begin{tabular}{llll}
\hline & pSS (n=I85) & Controls (n=I68) & $P$-value \\
\hline Physical function & $76.5 I \pm 23.10$ & $92.97 \pm I I .28$ & $<0.001$ \\
Role physical & $32.56 \pm 38.62$ & $92.56 \pm 19.83$ & $<0.001$ \\
Body pain & $74.51 \pm 23.55$ & $88.34 \pm 15.79$ & $<0.001$ \\
General health & $41.19 \pm 19.67$ & $69.95 \pm 16.05$ & $<0.001$ \\
Vitality & $55.16 \pm 15.35$ & $74.08 \pm 10.99$ & $<0.001$ \\
Social function & $69.12 \pm 21.93$ & $87.35 \pm 12.16$ & $<0.001$ \\
Role emotional & $42.88 \pm 45.43$ & $92.06 \pm 21.96$ & $<0.001$ \\
Mental health & $66.98 \pm 13.26$ & $78.43 \pm 8.53$ & $<0.001$ \\
Physical composite score & $52.80 \pm 19.4 I$ & $85.96 \pm 10.33$ & $<0.001$ \\
Mental composite score & $59.21 \pm 20.92$ & $82.98 \pm 9.85$ & $<0.001$ \\
\hline
\end{tabular}

Note: Data are presented as mean \pm SD.

Abbreviations: pSS, primary Sjögren's syndrome; SF-36, Short Form 36 health survey. fatigue correlated with all domains of the SF-36. Fatigue and pain are the most common extraglandular symptoms, ${ }^{35}$ and pain severity explained $14 \%$ of the variance in disability of daily activities in Korean patients with pSS. ${ }^{36}$ Our results were in line with previous studies reporting that pain is a major symptomatic problem related to low physical functioning and health outcomes. Therefore, systematic monitoring of pain treatment seems essential.

The results showed that pSS patients had a moderate level of disease activity, with a mean ESSDAI score of 9.56. Similarly, the mean ESSDAI score of pSS patients from multicenter clinics of France was 9.04. ${ }^{37}$ The comparison to age- and sex-matched healthy controls suggests that the disease-related symptoms of pSS patients are not attributed to the natural process of aging, but age was truly thought to play a crucial role in dysregulation of the neuroendocrine system. ${ }^{38} \mathrm{We}$ found that patient-perceived swallowing disorders were relatively common in pSS and increased with disease severity, which was uniquely associated with reduced QoL. Since eating and drinking form an important part of social interaction, dysphagic pSS patients often eat in seclusion because of shame. Also, they worry choking on their food or develop aspiration pneumonia. Frequent worries can decrease the QoL even more and aggravate anxiety. Therefore, health care professionals need to pay more attention to pSS patients who manifest swallowing symptoms and to strengthen the assessment of swallowing function of the pSS patients in clinical practice.

Notably, a significant difference was found in the marital status between the groups. The possible explanation is that women with pSS often suffer from vaginal dryness. Many pSS patients were unable to continue regular marital relationship due to sexual difficulties. Disease severity and disease duration may cause an increase in the divorce rate.

There are several limitations of this study. First, this study is cross-sectional in design, which does not allow for following up the progress of the examined variables during the course of the disease, and thus, we are unable to comment on the causal relationships. Second, it fails to make a distinction between men and women because of the great sex disparity. Third, all subjects were from a single hospital and the scales were all self-administered, which might give rise to possible biases of the outcomes. Even so, we still consider that our results may provide important insights into this complex process by identifying the associations between variables and QoL in pSS patients. 
Table 3 Correlations between disease activity, anxiety/depression, fatigue, pain, age, and QoL in pSS patients

\begin{tabular}{|c|c|c|c|c|c|c|c|c|c|c|c|c|}
\hline \multirow{2}{*}{$\begin{array}{l}\text { Domains } \\
\text { of SF-36 }\end{array}$} & \multicolumn{2}{|c|}{ ESSDAI } & \multicolumn{2}{|l|}{ VAS } & \multicolumn{2}{|c|}{ HADS-A } & \multicolumn{2}{|c|}{ HADS-D } & \multicolumn{2}{|l|}{ FSS } & \multicolumn{2}{|l|}{ Age } \\
\hline & $R$ & $P$-value & $R$ & $P$-value & $R$ & $P$-value & $R$ & $P$-value & $R$ & $P$-value & $R$ & $P$-value \\
\hline$\overline{P F}$ & -0.232 & $0.00 I^{* *}$ & -0.334 & $0.000^{* *}$ & -0.253 & $0.00 I^{* *}$ & -0.363 & $0.000^{* *}$ & -0.355 & 0.000 ** & -0.368 & $0.000 * *$ \\
\hline $\mathrm{RP}$ & -0.354 & $0.000 * *$ & -0.251 & $0.001 * *$ & -0.415 & $0.000 * *$ & -0.403 & $0.003^{* *}$ & -0.453 & $0.000 * *$ & -0.308 & $0.000 * *$ \\
\hline $\mathrm{BP}$ & -0.304 & $0.000 * *$ & -0.697 & $0.000^{* *}$ & -0.216 & $0.003^{* *}$ & -0.250 & $0.00 I^{* *}$ & -0.312 & $0.00 I^{* *}$ & $-0.08 \mathrm{I}$ & 0.270 \\
\hline $\mathrm{GH}$ & -0.361 & $0.000 * *$ & -0.250 & $0.001 * *$ & -0.495 & $0.000 * *$ & -0.480 & $0.000^{* *}$ & -0.420 & 0.000 ** & -0.203 & $0.005 * *$ \\
\hline VT & -0.274 & $0.000 * *$ & -0.274 & $0.000^{* *}$ & -0.463 & $0.000^{* *}$ & -0.497 & $0.000 * *$ & -0.542 & $0.000 * *$ & -0.27 I & $0.000 * *$ \\
\hline SF & -0.428 & $0.000 * *$ & -0.342 & $0.000 * *$ & -0.400 & $0.000 * *$ & -0.493 & 0.000 ** & -0.467 & 0.000 ** & -0.238 & $0.001 * *$ \\
\hline RE & -0.299 & $0.000 * *$ & -0.230 & $0.002^{* *}$ & -0.475 & $0.000^{* *}$ & -0.463 & $0.000^{* *}$ & -0.404 & $0.000 * *$ & -0.166 & $0.000 * *$ \\
\hline $\mathrm{MH}$ & -0.249 & $0.00 I^{* *}$ & -0.254 & $0.000^{* *}$ & -0.648 & $0.000 * *$ & -0.672 & $0.000^{* *}$ & -0.337 & $0.000 * *$ & -0.195 & $0.008 * *$ \\
\hline PCS & -0.287 & $0.000 * *$ & -0.413 & $0.000^{* *}$ & -0.461 & $0.000 * *$ & -0.493 & $0.000^{* *}$ & -0.388 & $0.000 * *$ & -0.294 & $0.000 * *$ \\
\hline MCS & -0.391 & $0.000 * *$ & -0.324 & $0.000 * *$ & -0.560 & $0.000 * *$ & -0.586 & $0.000 * *$ & -0.530 & $0.000 * *$ & -0.231 & $0.002 * *$ \\
\hline
\end{tabular}

Note: $* * P<0.01$.

Abbreviations: BP, bodily pain; ESSDAI, European League Against Rheumatism Sjögren's Syndrome Disease Activity Index; FSS, Fatigue Severity Scale; GH, general health; HADS-A, Hospital Anxiety Scale; HADS-D, Hospital Depression Scale; MCS, mental composite score; MH, mental health; PCS, physical composite score; PF, physical functioning; pSS, primary Sjögren's syndrome; QoL, quality of life; RE, role emotional; RP, role physical; SF, social functioning; SF-36, Short Form 36 health survey; VAS, visual analog scale; VT, vitality.

\section{Conclusion}

Patients with pSS had a considerable impaired QoL in comparison with the healthy subjects. pSS could have an unfavorable influence on the QoL of patients. Measuring QoL should be considered as a vital part of the comprehensive evaluation of the health status of pSS patients, which could contribute some valuable clues to improving the management of disease and treatment decisions.

\section{Acknowledgments}

This study was funded by the Chinese National Natural Science Foundation (Grant no 81671616 and 81471603), Jiangsu Provincial Commission of Health and Family Planning Foundation (Grant no H201317 and H201623), the

Table 4 Result of analysis of forward stepwise ordered linear regression models in SF-36

\begin{tabular}{|c|c|c|c|c|}
\hline Predictors & $\beta$ & SE & $P$-value & B $(95 \% \mathrm{Cl})$ \\
\hline \multicolumn{5}{|l|}{ MCS } \\
\hline Depression & -0.260 & 0.392 & 0.001 & $-1.309(-2.083,-0.535)$ \\
\hline FSS & -0.251 & 0.725 & 0.000 & $-3.053(-4.483,-1.623)$ \\
\hline $\begin{array}{l}\text { Impaired } \\
\text { swallowing }\end{array}$ & 0.171 & 0.068 & 0.004 & $0.198(0.064,0.332)$ \\
\hline ESSDAI & -0.181 & 0.160 & 0.001 & $-0.525(-0.916,-0.280)$ \\
\hline Anxiety & -0.165 & 0.411 & 0.037 & $-0.866(-1.677,-0.055)$ \\
\hline \multicolumn{5}{|l|}{ PCS } \\
\hline $\begin{array}{l}\text { Impaired } \\
\text { swallowing }\end{array}$ & 0.322 & 0.065 & 0.000 & $0.346(0.217,0.475)$ \\
\hline Depression & -0.327 & 0.282 & 0.000 & $-1.526(-2.082,-0.970)$ \\
\hline Pain & -0.260 & 0.444 & 0.000 & $-1.969(-2.846,-1.093)$ \\
\hline
\end{tabular}

Abbreviations: ESSDAI, European League Against Rheumatism Sjögren's Syndrome Disease Activity Index; FSS, Fatigue Severity Scale; MCS, mental composite score; PCS, physical composite score; SE, standard error; SF-36, Short Form 36 health survey.
Science Foundation of Nantong City (Grant no MS32015021, MS2201564, MS22016028, and MS22016019), and the Science and Technology Foundation of Nantong City (Grant no HS2014071 and HS2016003).

\section{Disclosure}

The authors report no conflicts of interest in this work.

\section{References}

1. Xiao F. Neuromyotonia as an unusual neurological complication of primary Sjögren's syndrome: case report and literature review. Clin Rheumatol. 2017;36(2):481-484.

2. Lackner A, Ficjan A, Stradner MH, et al. It's more than dryness and fatigue: The patient perspective on health-related quality of life in primary Sjögren's syndrome - a qualitative study. PLoS One. 2017;12(2): e1720562017-02-09.

3. Papageorgiou A, Voulgarelis M, Tzioufas AG. Clinical picture, outcome and predictive factors of lymphoma in Sjögren syndrome. Autoimmun Rev. 2015;14(7):641-649.

4. Brito-Zero NPB. Sjogren syndrome. Nat Rev Dis Primers. 2016;2: 16047.

5. Gupta S, Gupta N. Sjögren Syndrome and Pregnancy: A Literature Review. Perm J. 2017;21:16-047.

6. Cornec D, Devauchelle-Pensec V, Mariette X, et al. Severe healthrelated quality of life impairment in active primary Sjögren's syndrome and patient-reported outcomes: data from a large therapeutic trial. Arthritis Care Res. 2017;69(4):528-535.

7. Milin M, Cornec D, Chastaing M, et al. Sicca symptoms are associated with similar fatigue, anxiety, depression, and quality-of-life impairments in patients with and without primary Sjögren's syndrome. Joint Bone Spine. 2016;83(6):681-685.

8. Koh JH, Kwok SK, Lee J, et al. Pain, xerostomia, and younger age are major determinants of fatigue in Korean patients with primary Sjögren's syndrome: a cohort study. Scand J Rheumatol. 2017;46(1):49-55.

9. Karageorgas T, Fragioudaki S, Nezos A, Karaiskos D, Moutsopoulos HM, Mavragani CP. Fatigue in primary Sjögren's syndrome: clinical, laboratory, psychometric, and biologic associations. Arthritis Care Res. 2016;68(1):123-131.

10. Mandl T, Jørgensen TS, Skougaard M, Olsson P, Kristensen LE. Work disability in newly diagnosed patients with primary Sjögren syndrome. J Rheumatol. 2017;44(2):209-215. 
11. Koçer B, Tezcan ME, Batur HZ, et al. Cognition, depression, fatigue, and quality of life in primary Sjögren's syndrome: correlations. Brain Behav. 2016;6(12):e586.

12. Leung KC, Mcmillan AS, Wong MC, Leung WK, Mok MY, Lau CS. The efficacy of cevimeline hydrochloride in the treatment of xerostomia in Sjögren's syndrome in southern Chinese patients: a randomised double-blind, placebo-controlled crossover study. Clin Rheumatol. 2008;27(4):429-436.

13. Tanner K, Pierce JL, Merrill RM, Miller KL, Kendall KA, Roy N. The quality of life burden associated with voice disorders in Sjögren's syndrome. Ann Otol Rhinol Laryngol. 2015;124(9):721-727.

14. Abushakra M. Quality of Life, Coping and Depression in Systemic Lupus Erythematosus. Isr Med Assoc J. 2016;18(3-4):144-145.

15. El-Rabbat MS, Mahmoud NK, Gheita TA. Clinical significance of fibromyalgia syndrome in different rheumatic diseases: relation to disease activity and quality of life. Rheumatol Clin. 2017.

16. Jiang Q, Zhang H, Pang R, Chen J, Liu Z, Zhou X. Acupuncture for primary Sjögren syndrome (pSS) on symptomatic improvements: study protocol for a randomized controlled trial. BMC Complement Altern Med. 2017;17(1):61

17. Catanzaro J, Dinkel S. Sjögren's syndrome: the hidden disease. Medsurg Nurs. 2014;23(4):219-223.

18. Meijer JM, Meiners PM, Huddleston Slater JJ, et al. Health-related quality of life, employment and disability in patients with Sjogren's syndrome. Rheumatology. 2009;48(9):1077-1082.

19. Seror R, Bowman SJ, Brito-Zeron P, et al. EULAR Sjögren's syndrome disease activity index (ESSDAI): a user guide. RMD Open. 2015; 1(1):e22.

20. Seror R, Ravaud P, Mariette X, et al. EULAR Sjogren's Syndrome Patient Reported Index (ESSPRI): development of a consensus patient index for primary Sjogren's syndrome. Ann Rheum Dis. 2011;70(6):968-972.

21. Wang W, Chair SY, Thompson DR, Twinn SF. A psychometric evaluation of the Chinese version of the Hospital Anxiety and Depression Scale in patients with coronary heart disease. J Clin Nurs. 2009;18(17): 2436-2443.

22. Dassouki T, Benatti FB, Pinto AJ, et al. Objectively measured physical activity and its influence on physical capacity and clinical parameters in patients with primary Sjögren's syndrome. Lupus. 2017;26(7): 690-697.

23. Ware JE, Gandek B, Kosinski M, et al. The equivalence of SF-36 summary health scores estimated using standard and country-specific algorithms in 10 countries: results from the IQOLA project. International Quality of Life Assessment. J Clin Epidemiol. 1998;51(11):1167-1170.

24. Lam CL, Gandek B, Ren XS, Chan MS. Tests of scaling assumptions and construct validity of the Chinese (HK) version of the SF-36 health survey. J Clin Epidemiol. 1998;51(11):1139-1147.
25. Lam CL, Tse EY, Gandek B, Fong DY. The SF-36 summary scales were valid, reliable, and equivalent in a Chinese population. $J$ Clin Epidemiol. 2005;58(8):815-822.

26. Li L, Wang HM, Shen Y. Chinese SF-36 health survey: translation, cultural adaptation, validation, and normalisation. J Epidemiol Community Health. 2003;57(4):259-263.

27. Krupp LB, Larocca NG, Muir-Nash J, Steinberg AD. The fatigue severity scale. Application to patients with multiple sclerosis and systemic lupus erythematosus. Arch Neurol. 1989;46(10):1121-1123.

28. Sokka T, Kankainen A, Hannonen P. Scores for functional disability in patients with rheumatoid arthritis are correlated at higher levels with pain scores than with radiographic scores. Arthritis Rheum. 2000; 43(2):386-389.

29. Pierce JL, Tanner K, Merrill RM, Miller KL, Kendall KA, Roy N. Swallowing disorders in Sjögren's syndrome: prevalence, risk factors, and effects on quality of life. Dysphagia. 2016;31(1):49-59.

30. Enger TB, Palm $\varnothing$, Garen T, Sandvik L, Jensen JL. Oral distress in primary Sjögren's syndrome: implications for health-related quality of life. Eur J Oral Sci. 2011;119(6):474-480.

31. Ibn Yacoub Y, Rostom S, Laatiris A, Hajjaj-Hassouni N. Primary Sjögren's syndrome in Moroccan patients: characteristics, fatigue and quality of life. Rheumatol Int. 2012;32(9):2637-2643.

32. Segal B, Bowman SJ, Fox PC, et al. Primary Sjögren's syndrome: health experiences and predictors of health quality among patients in the United States. Health Qual Life Outcomes. 2009;7(46):1.

33. Cho HJ, Yoo JJ, Yun CY, et al. The EULAR Sjogren's syndrome patient reported index as an independent determinant of health-related quality of life in primary Sjogren's syndrome patients: in comparison with nonSjogren's sicca patients. Rheumatology. 2013;52(12):2208-2217.

34. Westhoff G, Dörner T, Zink A. Fatigue and depression predict physician visits and work disability in women with primary Sjögren's syndrome: results from a cohort study. Rheumatology. 2012;51(2):262-269.

35. Segal B, Thomas W, Rogers T, et al. Prevalence, severity, and predictors of fatigue in subjects with primary Sjögren's syndrome. Arthritis Rheum. 2008;59(12):1780-1787.

36. Shim E-J, Hahm B-J, Go DJ, et al. Modeling quality of life in patients with rheumatic diseases: the role of pain catastrophizing, fear-avoidance beliefs, physical disability, and depression. Disabil Rehabil. 2018; 40(13):1509-1516.

37. Seror R, Ravaud P, Bowman SJ, et al. EULAR Sjogren's syndrome disease activity index: development of a consensus systemic disease activity index for primary Sjogren's syndrome. Ann Rheum Dis. 2010; 69(6):1103-1109.

38. Oxenkrug GF. Metabolic syndrome, age-associated neuroendocrine disorders, and dysregulation of tryptophan-kynurenine metabolism. Ann N Y Acad Sci. 2010;1199(1):1-14.
Patient Preference and Adherence

\section{Publish your work in this journal}

Patient Preference and Adherence is an international, peer-reviewed, open access journal that focuses on the growing importance of patient preference and adherence throughout the therapeutic continuum. Patient satisfaction, acceptability, quality of life, compliance, persistence and their role in developing new therapeutic modalities and compounds to optimize

\section{Dovepress}

clinical outcomes for existing disease states are major areas of interest for the journal. This journal has been accepted for indexing on PubMed Central. The manuscript management system is completely online and includes a very quick and fair peer-review system, which is all easy to use. Visit http://www. dovepress.com/testimonials.php to read real quotes from published authors. 